\title{
Using a filming protocol to improve video- instructed cardiopulmonary resuscitation
}

\author{
Omer Perry $^{\mathrm{a}}$, Oren Wacht ${ }^{\mathrm{b}, \mathrm{c}}$, Eli Jaffe ${ }^{\mathrm{b}, \mathrm{c}}$, Zilla Sinuany-Stern ${ }^{\mathrm{a}}$ and Yuval Bitan ${ }^{\mathrm{a}, *}$ \\ ${ }^{a}$ Department of Industrial Engineering and Management, Ben-Gurion University of the Negev, \\ Beer-Sheva, Israel \\ ${ }^{\mathrm{b}}$ Department of Emergency Medicine, Ben-Gurion University of the Negev, Beer-Sheva, Israel \\ ${ }^{\mathrm{c}}$ Magen-David-Adom (Israel National Emergency Medical Service), Israel
}

Received 31 October 2019

Accepted 20 November 2019

\begin{abstract}
.
BACKGROUND: Video communications during cardiopulmonary resuscitation (CPR) can improve the quality of information exchange between a bystander performing CPR and an emergency medical dispatcher (EMD).

OBJECTIVE: To improve chest compression effectiveness, a filming protocol instructing video camera placements around a patient was developed. This study measured whether the filming protocol increased chest compressions' effectiveness.

METHODS: A simulation study was conducted comparing CPR effectiveness under three conditions: telephone-instructed, video-instructed, and video-instructed with the filming protocol. Twenty-five emergency medical technicians acted as EMDsin the three conditions. A mannequin measured five factors that determined the effectiveness of the chest compressions.

RESULTS: Compared with telephone-instructed CPR, the filming protocol improved the proportion of time in which the bystander's hands were in the correct position during chest compressions. Compared with video-instructed CPR, the filming protocol improved both the proportion of time in which the chest was fully released after each compression and the proportion of time in which the compressions were conducted with an appropriate rhythm. The depth and rate of compressions did not improve in the filming protocol condition.

CONCLUSIONS: Video-instructed CPR with the filming protocol improves CPR effectiveness compared to telephone- and video-instructed CPR. Detailed implementation can improve new technology introduction.
\end{abstract}

Keywords: Emergency medical dispatcher, cardiac arrest, telephone cardiopulmonary resuscitation, video cardiopulmonary resuscitation, filming protocol

\section{Introduction}

Out-of-hospital cardiac arrest (OHCA) is one of the leading causes of mortality around the world [1,2]. Its identification and the immediate application of high-quality cardiopulmonary resuscitation (CPR) can

\footnotetext{
${ }^{*}$ Corresponding author: Yuval Bitan, Department of Industrial Engineering and Management, Ben-Gurion University of the Negev, P.O. box 653, Beer-Sheva, Israel. Tel.: +972 54 6543210; E-mail: yuval@bitan.net.
}

0928-7329/20/\$35.00 (c) 2020 - IOS Press and the authors. All rights reserved

This article is published online with Open Access and distributed under the terms of the Creative Commons Attribution NonCommercial License (CC BY-NC 4.0). 
significantly improve survival rates [3-8]. Emergency medical dispatchers (EMDs) instruct bystanders at OHCA events to perform chest compressions until the arrival of an Emergency Medical Services (EMS) team. However, performance of high-quality chest compressions remains low [9].

Some EMD call centers apply a live video communications channel with the bystander to improve the quality of information provided to the dispatcher [10-15]. To further improve chest compression effectiveness using live video communication, a filming protocol for the EMD was developed. With this protocol EMDs guide the bystander to position the camera at specific filming angles around the patient. These filming angles allow the dispatcher to measure chest compressions effectiveness, and to provide the bystander with relevant feedback to improve resuscitation efforts. This study aims to measure the effect of the filming protocol on the effectiveness of chest compression performance.

\section{Methods}

To improve the video communication channel, we developed a filming protocol that defines three filming angles around the patient (Fig. 1): (1) In front of the bystander; (2) Above the patient's legs high; and (3) In front of the patient's legs - low.

Instructions on where to position the camera to capture these filming angles were merged with current telephone-instructed CPR protocol used by Magen-David-Adom (Israel National EMS) EMDs for OHCA (marked in red in Fig. 2).

The independent variables were three communication protocols as between-subjects conditions: (1) Telephone-instructed CPR protocol without video communications; (2) Video-instructed CPR - using the telephone-instructed CPR protocol with the addition of a video communication channel; (3) Video-instructed with the filming protocol - telephone-instructed CPR protocol with specific filming angles instructions.

The dependent variables were five chest compression effectiveness measurements: (1) Compressions depth, measured in $\mathrm{mm}$ (target: 50-60 mm) [9]; (2) Chest compression rhythm, measured in beats per minute (BPM) (target: 100-120 BPM) [9]; (3) Percentage of time the bystander positioned their hands in the centre of the patient's chest [9]; (4) Percentage of time the chest compressions were performed with the correct rhythm [9]; (5) Percentage of time the bystander fully released the chest after each compression [24]. We used a Laerdel QCPR ${ }^{\circledR}$ mannequin to simulate the chest compressions [16], and measure these factors. Placing of one hand over the other is also considered an important effectiveness measurement [17], but it could not be measured by the mannequin. For the first and second measurements the Mann-Whitney U test was used for comparison between the three conditions. For the three other measurements the (chi) test was used. Results are reported as percentages with the addition of 95\% confidence intervals $(95 \% \mathrm{CI})$ for the proportion means. Results were considered statistically significant at the $5 \%$ level $(p<0.05)$.

The simulation was conducted in two rooms - one for the dispatcher and one for the bystander. The dispatcher's room was equipped with the relevant communication protocol, a mobile phone for audio communication, and an Apple iPad ${ }^{\circledR}$ for video communication (for both video conditions). The bystanders' room was equipped with a Laerdal $\mathrm{QCPR}^{\circledR}$ mannequin, a screen that presented the patient emergency scenario, a mobile phone for audio communications, and a GO-PRO-5 ${ }^{\circledR}$ camera, which streamed live video to the other room (for both video conditions).

Each simulation scenario began in the bystander room by playing a short OHCA video that included a male patient [18] who was unconscious and not breathing normally [1]. The bystander used the mobile telephone to report the emergency and to receive instructions from the dispatcher. 


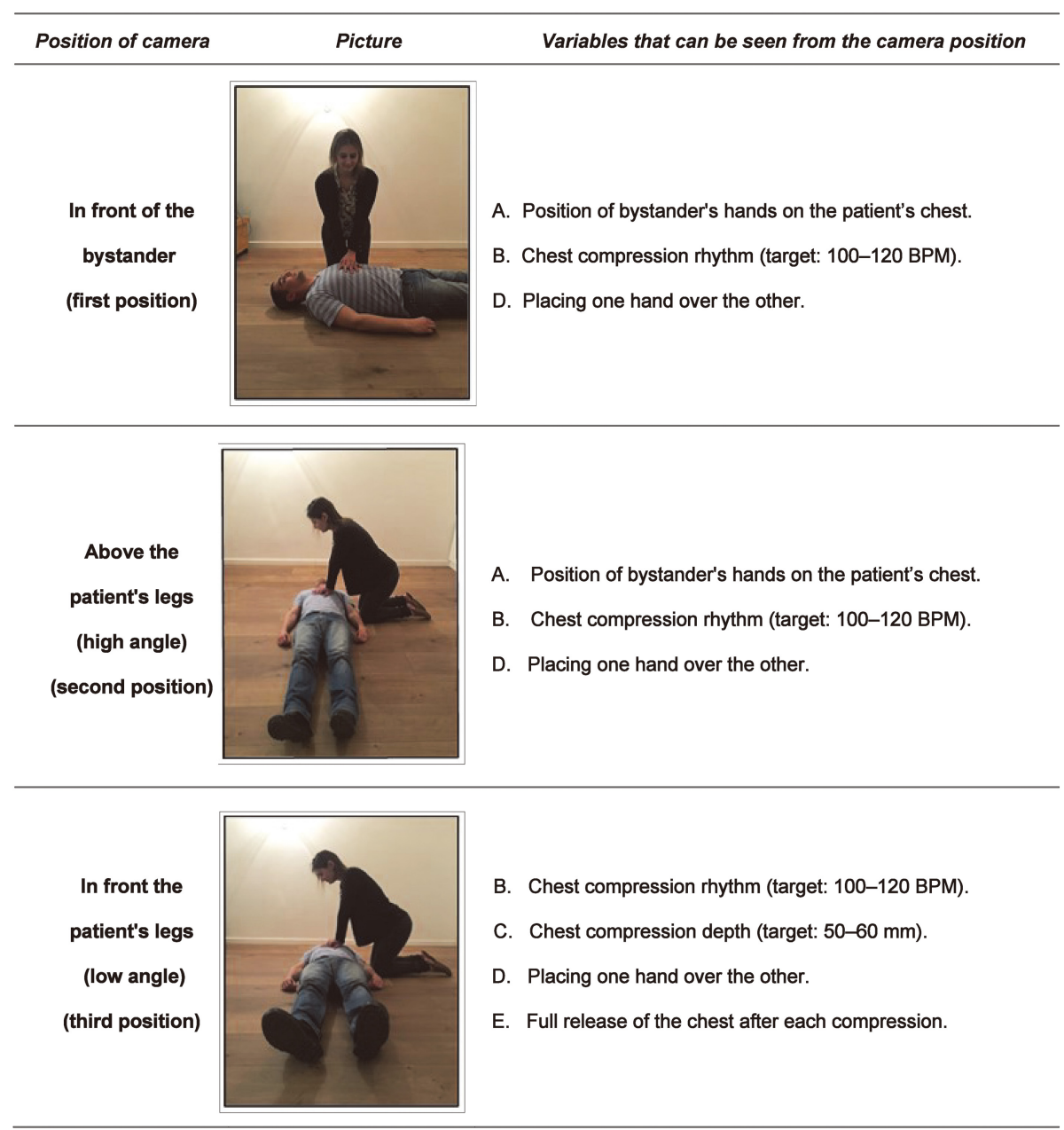

Fig. 1. Camera positions for the video-instructed CPR with filming protocol.

Every simulation session included two scenarios: The first featured the telephone-instruct CPR condition with one bystander and a dispatcher, and the second scenario included one of the two video conditions with two bystanders and a dispatcher.

The dispatchers in the simulation were experienced EMTs (Emergency Medical Technicians) that were in their third-year of paramedicine studies, trained at performing CPR. Prior to the simulation, all EMTs had a short training on the use of the telephone-instructed CPR protocol and the filming protocol. Participants in the role of bystanders were students without previous experience in performing chest compressions. Ethics approval was given by the Human-Subjects Research Committee of Ben-Gurion University (1507-1).

\section{Results}

Overall 25 simulation sessions were conducted, which included 49 scenarios - 17 scenarios under the telephone-instructed CPR condition, 14 under the video-instructed CPR condition, and 18 under the video-instructed CPR with filming protocol condition. The results are summarized in Table 1. 


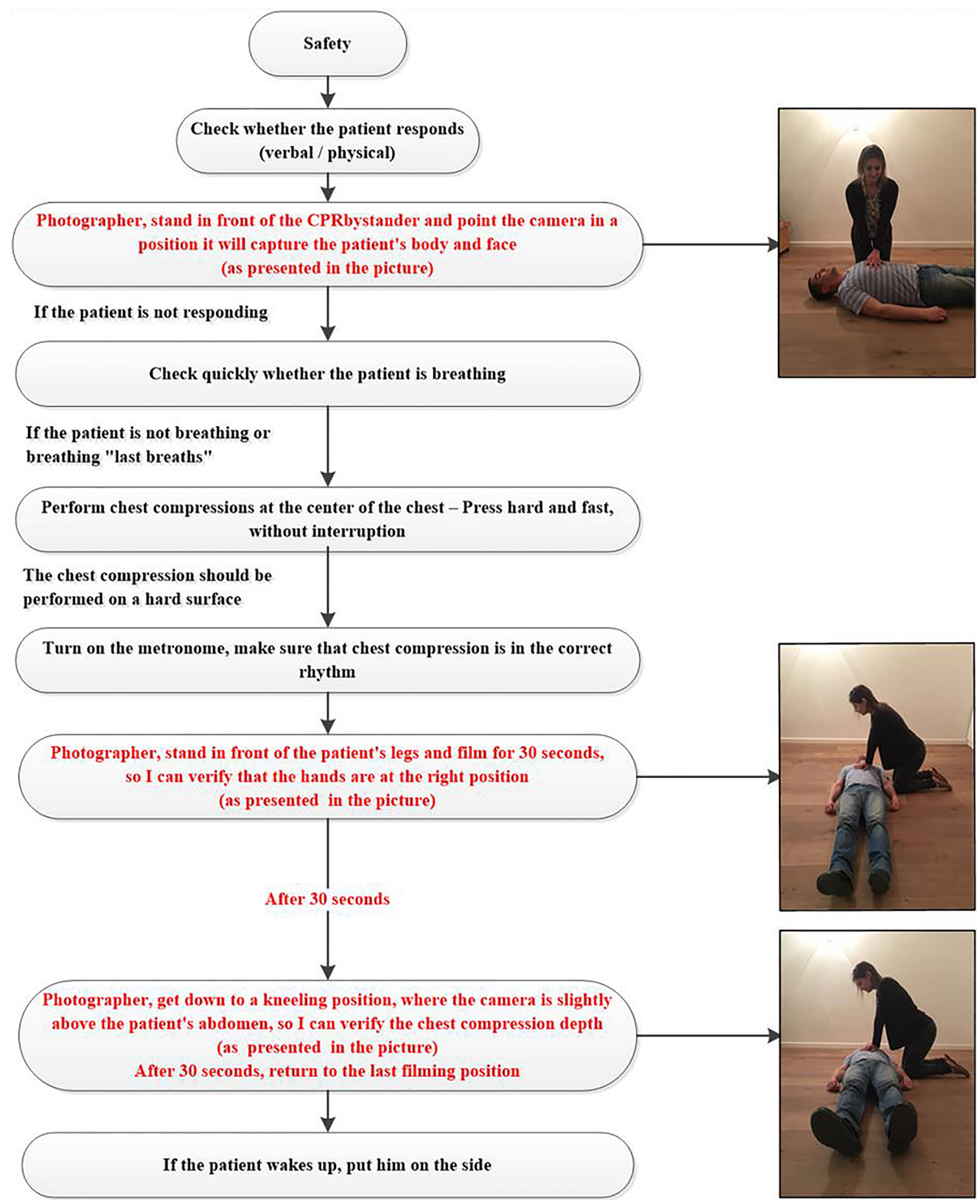

Fig. 2. Video-instructed CPR with filming protocol. 
Table 1

Results of the five measurements in the three experimental conditions

\begin{tabular}{|c|c|c|c|}
\hline & $\begin{array}{l}\text { Telephone-instructed CPR } \\
(n=17)\end{array}$ & $\begin{array}{l}\text { Video-instructed CPR } \\
(n=14)\end{array}$ & $\begin{array}{l}\text { Video-instructed CPR with } \\
\text { filming protocol }(n=18)\end{array}$ \\
\hline Depth of compressions & $26.00[\mathrm{~mm}]$ & $\begin{array}{l}25.00[\mathrm{~mm}] \\
p=0.28 \text { compared with } \\
\text { t-CPR }\end{array}$ & $\begin{array}{l}26.00[\mathrm{~mm}] \\
p=0.37 \text { compared with } \\
\text { t-CPR } \\
p=0.92 \text { compared with } \\
\text { v-CPR }\end{array}$ \\
\hline Rhythm & $103.00[\mathrm{BPM}]$ & $\begin{array}{l}100.00[\mathrm{BPM}] \\
p=0.95 \text { compared with } \\
\text { t-CPR }\end{array}$ & $\begin{array}{l}107.50[\mathrm{BPM}] \\
p=0.25 \text { compared with } \\
\text { t-CPR } \\
p=0.16 \text { compared with } \\
\mathrm{v}-\mathrm{CPR}\end{array}$ \\
\hline $\begin{array}{l}\text { Hands placed correctly in the } \\
\text { centre of patient's chest }\end{array}$ & $62.94 \%$ & $\begin{array}{l}71.36 \% \\
p<0.05 \text { compared with } \\
\text { t-CPR* }\end{array}$ & $\begin{array}{l}73.17 \% \\
p<0.05 \text { compared with } \\
\text { t-CPR } * \\
p=0.20 \text { compared with } \\
\text { v-CPR }\end{array}$ \\
\hline $\begin{array}{l}\text { Percentage of time chest com- } \\
\text { pressions were done with cor- } \\
\text { rect rhythm }\end{array}$ & $54.18 \%$ & $\begin{array}{l}47.29 \% \\
p<0.05 \text { compared with } \\
\text { t-CPR* }\end{array}$ & $\begin{array}{l}53.61 \% \\
p=0.70 \text { compared with } \\
\text { t-CPR } \\
p<0.05 \text { compared with } \\
\text { v-CPR* }\end{array}$ \\
\hline $\begin{array}{l}\text { Full release of the chest after } \\
\text { each compression }\end{array}$ & $74.65 \%$ & $\begin{array}{l}61 \% \\
p<0.05 \text { compared with } \\
\text { t-CPR* }\end{array}$ & $\begin{array}{l}81.56 \% \\
p=0.43 \text { compared with } \\
\text { t-CPR } \\
p<0.05 \text { compared with } \\
\text { v-CPR } *\end{array}$ \\
\hline
\end{tabular}

Values are presented as percentages or quantitatively. Percentages were compared using a test. Quantitative variables were compared using a Mann-Whitney U test. Significant differences are marked with *.

\subsection{Compressions depth}

Median compressions depth was the same in the video with filming protocol condition [2.60 (2.28, $4.60) \mathrm{mm}]$ and in the telephone-instructed condition $[2.60,(1.80,3.60) \mathrm{mm}](p=0.37)$. The median depth of compressions was lowest in the video-instructed condition $[2.50(2.25,4.35) \mathrm{mm}]$, but was not significantly different compared to the telephone-instructed condition $(p=0.28)$ and the videoinstructed condition (0.92).

\subsection{Chest compression rhythm}

The fastest chest compressions rhythm was observed in the video with the filming protocol condition [107.50 (98.25, 114.50) BPM], but it was not significantly different from the video-instructed condition $[100.00(88.50,112.50) \mathrm{BPM} ; p=0.16]$ or the telephone-instructed condition $[103.00,(70.50,112.00)$ BMP; $p=0.25]$.

\subsection{Percentage of time the bystander's hands were placed correctly}

Correct position of the hands on the patient's chest was observed in $71.36 \%$ (95\% CI 48.24\%-94.48\%) of the scenarios in the video-instructed condition, and in $73.17 \%$ (95\% CI 53.94\%-92.39\%) of the scenarios in the video with the filming protocol condition; thus, these conditions were not significantly 
different from each other $(p=0.20)$. In contrast, correct position of the hands was observed in only $62.94 \%$ (95\% CI 40.94\%-84.93\%) of the scenarios in the telephone-instructed condition, which was significantly different from both video conditions ( $p<0.05$ for each comparison).

\subsection{Percentage of time chest compressions were done with the correct rhythm}

The percentage of time in which the bystander administered CPR with the correct rhythm (100120 BMP) was significantly lower in the video-instructed condition $(47.29 \%, 95 \%$ CI 30.17\%-64.40\%) than in either the telephone-instructed condition $(54.18 \%, 95 \%$ CI $34.89 \%-73.49 \%, p<0.05)$ or the video with filming protocol condition $(53.61 \%$, 95\% CI 35.70\%-71.52\%, $p<0.05)$, which were not significantly different from each other $(p=0.07)$.

\subsection{Percentage of time the bystander fully released the chest after each compression}

The video-instructed condition demonstrated the lowest proportion of time in which the chest was fully released $(61.00 \%$, CI $39.29 \%-82.71 \%)$, and it was significantly lower than in the telephoneinstructed condition $(74.65 \%$, CI $56.21 \%-93.09 \%, p<0.05)$ or in the video with filming protocol condition, which demonstrated the highest proportion of time $(81.56 \%$, CI $66.68 \%-96.43 \%, p<0.05)$. No significant difference was found between the video-instructed condition and the video with filming protocol condition $(p=0.43)$.

\section{Discussion}

Information exchange in the telephone-instructed CPR protocol is based only on audio information. Adding the live video communications channel provides additional information that includes visual information. The current study found that designing the way the additional video information is acquired might improve information quality. Thus, the filming protocol might be an important part of an improved video communications channel, improving patient outcomes. From every camera angle, bystanders can focus on a specific valuable piece of information (e.g., measurements for the evaluation of the effectiveness of chest compressions) for a limited duration. And thus, all the measurements that are important for chest compression effectiveness [9,19-25] can be evaluated by the EMD while a bystander performs the chest compressions.

The study found that the use of live video communications channel with the filming protocol improved the effectiveness of chest compressions compared to the video communications channel with the telephone-instructed CPR protocol, which showed a decrease in the effectiveness of chest compressions. However, in the current study, one measurement did not show improvement due to the addition of the filming protocol. In line with past results $[12,19]$, the depth of compressions remained low and out of the required boundaries of 50-60 mmin all the experimental conditions. The fact that the depth of compressions can be seen from only one angle could be the reason for these results. Prioritization of the angle order (e.g., rearranging the order so that the low angle position is first) and a longer duration for this specific angle might improve these results. Future studies should focus on optimizing the filming protocol and on improving the depth of compressions.

The filming protocol improves some of the factors that affect the effectiveness of chest compressions performed by a bystander in an OHCA event, and should be part of the future medical dispatcher's methods. These findings suggest that in order to get the most out of new technology, healthcare providers need to pay special attention to its implementation in the field. 


\section{Acknowledgments}

This work was supported by a research grant from the Israel National Institute for Health Policy Research [grant number 153/2016/R]. The authors would like to thank Eldar Muzikansky and Yuval De Levy for their support in conducting the experiments.

\section{Conflict of interest}

None to report.

\section{References}

[1] Hazinski M, Shuster M, Donnino M, Travers A, Samson R, Schexnayder S, et al. Highlights of the 2015 American Heart Association - Guidelines Update for CPR and ECG. Am Hear Assoc. 2015; 1-36.

[2] Perkins GD, Handley AJ, Koster RW, Castrén M, Smyth MA, Olasveengen T, et al. European Resuscitation Council Guidelines for Resuscitation 2015. Section 2. Adult basic life support and automated external defibrillation. Resuscitation. 2015; 95: 81-99.

[3] Hasselqvist-Ax I, Riva G, Herlitz J, Rosenqvist M, Hollenberg J, Nordberg P, et al. Early Cardiopulmonary Resuscitation in Out-of-Hospital Cardiac Arrest. N Engl J Med. 2015; 372(24): 2307-15.

[4] Wissenberg M, Lippert FK, Folke F, Weeke P, Hansen CM, Christensen EF, et al. Association of national initiatives to improve cardiac arrest management with rates of bystander intervention and patient survival after out-of-hospital cardiac arrest. JAMA - J Am Med Assoc. 2013; 310(13): 1377-84.

[5] Sasson C, Rogers MAM, Dahl J, Kellermann AL. Predictors of Survival From Out-of-Hospital Cardiac Arrest: A Systematic Review and Meta-Analysis. Circ Cardiovasc Qual Outcomes. 2010; 3(1): 63-81.

[6] Bobrow BJ, Spaite DW, Vadeboncoeur TF, Hu C, Mullins T, Tormala W, et al. Implementation of a Regional Telephone Cardiopulmonary Resuscitation Program and Outcomes After Out-of-Hospital Cardiac Arrest. JAMA Cardiol. 2016; 1(3): 294-302.

[7] Rea TD, Eisenberg MS, Culley LL, Becker L. Dispatcher-Assisted Cardiopulmonary Resuscitation and Survival in Cardiac Arrest. Circulation. 2001; 104(21): 2513-6.

[8] Hollenberg J, Svensson L, Rosenqvist M. Out-of-hospital cardiac arrest: 10 years of progress in research and treatment. J Intern Med. 2013; 273(6): 572-83.

[9] Ghuysen A, Collas D, Stipulante S, Donneau AF, Hartstein G, Hosmans T, et al. Dispatcher-assisted telephone cardiopulmonary resuscitation using a French-language compression-only protocol in volunteers with or without prior life support training: A randomized trial. Resuscitation. 2011; 82(1): 57-63.

[10] Pettinari CJ. 'Your ears become your eyes': managing the absence of visibility in NHS Direct, 2001.

[11] Johnsen E, Bolle SR. TO SEE OR NOT TO SEE-Better dispatcher-assisted CPR with video-calls? A qualitative study based on simulated trials. Resuscitation. 2008; 78(3): 320-6.

[12] Bolle SR, Scholl J, Gilbert M. Can video mobile phones improve CPR quality when used for dispatcher assistance during simulated cardiac arrest? Acta Anaesthesiol Scand. 2009; 53(1): 116-20.

[13] Yang CW, Wang HC, Chiang WC, Chang WT, Yen ZS, Chen SY, et al. Impact of adding video communication to dispatch instructions on the quality of rescue breathing in simulated cardiac arrests-A randomized controlled study. Resuscitation. 2008; 78(3): 327-32.

[14] Bolle SR, Johnsen E, Gilbert M. Video calls for dispatcher-assisted cardiopulmonary resuscitation can improve the confidence of lay rescuers - surveys after simulated cardiac arrest. J Telemed Telecare. 2011; 17(2): 88-92.

[15] Melbye S, Hotvedt M, Bolle SR. Mobile videoconferencing for enhanced emergency medical communication - a shot in the dark or a walk in the park? - A simulation study. Scand J Trauma Resusc Emerg Med. 2014; 22(1): 1-7.

[16] April R. Little Ann QCPR Scroring System [Internet]. 2015; Available from: https//laerdal.egain.cloud/system/templates/ selfservice/Laerdal_Customer/help/customer/locale/en-US/portal/404700000001006/content-version/Prod-9423/Prod217645/How-is-QCPR-Scoring-calculated.

[17] Baubin M, Kollmitzer J, Pomaroli A, Kraincuk P, Kranzl A, Sumann G, et al. Force distribution across the heel of the hand during simulated manual chest compression. Resuscitation. 1997; 35(3): 259-63.

[18] Bielecki JM, Wong J, Mitsakakis N, Shah PS, Krahn MD, Rac VE. Disparities in a provision of in-hospital post-arrest interventions for out-of-hospital cardiac arrest (OHCA) in the elderly population - protocol for a systematic review. Syst Rev. 2016; 1-9. 
[19] Yang CW, Wang HC, Chiang WC, Hsu CW, Chang WT, Yen ZS, et al. Interactive video instruction improves the quality of dispatcher-assisted chest compression-only cardiopulmonary resuscitation in simulated cardiac arrests. Crit Care Med. 2009; 37(2): 490-5.

[20] Yang Z, Li H, Yu T, Chen C, Xu J, Chu Y, et al. Quality of chest compressions during compression-only cardiopulmonary resuscitation: a comparative analysis following the 2005 and 2010 American Heart Association guidelines. Am J Emerg Med. 2013; 32: 50-4.

[21] Woollard M, Smith A, Whitfield R, Chamberlain D, West R, Newcombe R, et al. To blow or not to blow: A randomised controlled trial of compression-only and standard telephone CPR instructions in simulated cardiac arrest. Resuscitation. 2003; 59(1): 123-31.

[22] Vadeboncoeur T, Stolz U, Panchal A, Silver A, Venuti M, Tobin J, et al. Chest compression depth and survival in out-ofhospital cardiac arrest. Resuscitation. 2014; 85(2): 182-8.

[23] Nishiyama C, Iwami T, Kawamura T, Ando M, Yonemoto N, Hiraide A, et al. Quality of chest compressions during continuous CPR; comparison between chest compression-only CPR and conventional CPR. Resuscitation. 2010; 81(9): 1152-5.

[24] Mirza M, Brown TB, Saini D, Pepper TL, Nandigam HK, Kaza N, et al. Instructions to "push as hard as you can" improve average chest compression depth in dispatcher-assisted cardiopulmonary resuscitation. Resuscitation. 2008; 79(1): 97-102.

[25] Roh YS, Lim EJ. Factors influencing quality of chest compression depth in nursing students. Int J Nurs Pract. 2013; 19(6): 591-5. 